\title{
Upregulation of suppressor of cytokine signaling 3 ameliorates spinal degenerative disease in adolescents by mediating leptin and tumor necrosis factor- $\alpha$ levels
}

\author{
XIAO-MING TANG ${ }^{*}$, JIAN DAI ${ }^{*}$ and HAI-LANG SUN \\ Department of Orthopedics, The Affiliated Huai'an No. 1 People's Hospital of Nanjing Medical University, \\ Huai'an, Jiangsu 223300, P.R. China
}

Received April 8, 2018; Accepted January 25, 2019

DOI: $10.3892 /$ etm.2019.7786

\begin{abstract}
Spinal degenerative changes may occur following the rapid growth observed in adolescents, causing a reduced quality of life. The suppressor of cytokine signaling (SOCS) is involved in various degenerative diseases. The current study recruited adolescents with spinal degenerative disease (SDD) to identify the effect of SOCS-3 on leptin and tumor necrosis factor- $\alpha$ (TNF- $\alpha)$ levels in this disorder. From January 2010 to January 2016, 120 adolescents (aged 14 to 25) were enrolled in the current study, with 68 diagnosed with SDD and the remaining 52 treated as controls. Nucleus pulposus cells (NPCs) were extracted and cultured in vitro. TNF- $\alpha$ levels in NPCs were determined using flow cytometry. Degenerative NPCs were then transfected with pCR3.1-SOCS-3 and ELISA was performed to determined TNF- $\alpha$ and leptin levels. RT-qPCR was performed to measure the mRNA level of SOCS-3 and leptin in NPCs and western blotting was utilized to detect the protein level of leptin and the extent of leptin receptor phosphorylation. The results revealed that TNF- $\alpha$ levels in degenerative NPCs were higher than those in normal NPCs. The overexpression of SOCS-3 reduced levels of TNF- $\alpha$ and leptin in degenerative NPCs. In addition, the upregulation of leptin increased SOCS-3 levels in a concentration-dependent manner. Furthermore, the expression of the leptin receptor and phosphorylated leptin receptor gradually decreased with increasing leptin concentrations and the level of phosphorylated leptin receptor negatively correlated with
\end{abstract}

Correspondence to: Dr Hai-Lang Sun, Department of Orthopedics, The Affiliated Huai'an No. 1 People's Hospital of Nanjing Medical University, Huanghe West Road 1, Huai'an, Jiangsu 223300, P.R. China

E-mail: sunhai_lang@126.com

*Contributed equally

Key words: suppressor of cytokine signaling 3, spinal degenerative disease, adolescent, leptin, tumor necrosis factor- $\alpha$, small interfering RNA, induction, inhibition
SOCS-3 expression. The inductive effect of leptin on the level of SOCS-3 and the inhibitory effect of SOCS-3 on the activity of leptin were identified. The current study demonstrated that SOCS-3 reduces leptin and TNF- $\alpha$ levels in degenerative NPCs from adolescents, indicating its potential role in the development of novel SDD therapies.

\section{Introduction}

Degenerative changes frequently occur in the spine and may present with or without back pain (1). More specifically, spinal degeneration is a disorder with an increasing frequency as a result of aging populations (2). Degenerative changes in intervertebral discs commonly occur in the third and second decade of life in females and males, respectively (3). In comparison with the dorsal spine, the cervical and lumbar spine are more commonly affected by degeneration (4). Patients suffering from spinal degenerative disease (SDD) experience back pain, radicular pain and stiffness, resulting in a reduced ability to work and a lower quality of life (5). Previous studies have revealed the prevalence of SDD in adolescents, which is associated with active participation in sports (6-8). The role of inflammatory cytokines in spinal diseases has been increasingly documented. Omair et al (9) have revealed that inflammation exacerbates the severity of lumbar disc degeneration, pain and disability. In addition, spinal cord injury induces inflammation via the activation of innate immune responses, causing axonal degeneration, demyelination and neuronal death (10). Furthermore, it has been demonstrated that the suppressor of cytokine signaling-3 (SOCS-3) serves as a potent regulator of inflammation (11) and spinal disease (12). Based on these previous findings, the current study hypothesized that SOCS-3 may have a potential role in SDD.

SOCS-3, a member of the SOCS protein family, combines with glycoprotein 130 or with Janus Kinase (JAK) 1 and JAK2 to suppress signal transduction (13). Osuka et al (14) reported that SOCS-3 molecules may serve as modulators in the degeneration of chondrocytes within herniated discs by negatively regulating the expression of JAK1 and signal transducer and activator of transcription 3 (STAT3). A previous study also demonstrated that enhanced SOCS-3 inhibits proliferation and inflammation in osteoarthritis (15), 
a degenerative joint disorder (16). In addition, SOCS-3 is a pivotal feedback inhibitor of leptin, while leptin induces the expression of SOCS-3 $(17,18)$. Leptin is a $16 \mathrm{kDa}$ hormone and a product of the " $o b$ " gene, which is generated by white adipocytes and regulates the intake of food and the balance of energy $(19,20)$. Leptin is also a direct regulator of bone growth, inducing osteoblast proliferation, collagen synthesis and bone mineralization (21). In addition, tumor necrosis factor- $\alpha$ (TNF- $\alpha$ ) is a prominent regulator of disc degeneration and lower back pain, exacerbating inflammatory processes (22). SOCS-3 regulates proinflammatory TNF- $\alpha$ signal transduction by suppressing TNF- $\alpha$-mediated signaling in hepatitis $\mathrm{C}$ virus infection (23). The current study assessed the effect of SOCS-3 on SDD in adolescents, as well as its involvement in regulating leptin and $\mathrm{TNF}-\alpha$. The results revealed that SOCS-3 protects degenerative nucleus pulposus cells (NPCs) against SDD in adolescents by modulating leptin and TNF- $\alpha$.

\section{Materials and methods}

Ethics statement. The experimental protocols of the present study were approved by the Ethical Committee of the Affiliated Huai'an No.1 People's Hospital of Nanjing Medical University (Huai'an, China). All patients provided signed informed consent.

Patients. From January 2010 to January 2016, 120 adolescents (age range, 14-25 years) admitted to The Affiliated Huai'an No.1 People's Hospital of Nanjing Medical University were selected for participation in the current study. X-ray and magnetic resonance imaging (MRI) scans were performed. The inclusion criteria were as follows: Patients > 20 years; patients that were diagnosed with SDD based on the following: Cervical spondylosis, cervical spinal stenosis, ossification of the posterior longitudinal ligament of the cervical vertebra, posterior longitudinal ligament ossification of the cervical vertebra, thoracic disc herniation, thoracic spinal stenosis, ossification of the posterior longitudinal ligament of the thoracic vertebra, lumbar disc herniation, lumbar spinal stenosis, non-traumatic spinal instability or spondylolisthesis. The exclusion criteria were as follows: Patients with cognitive or communication disorders that were unable to complete the scale and express their feelings clearly; patients who are critically ill or have other chronic diseases that lead to life dysfunction; patients that had experienced trauma, including spinal fracture within 3 months; and patients diagnosed with spinal neoplasms, tuberculosis or ankylosing spondylitis. According to Gries' scoring standard (24), a total of 68 patients (46 males and 22 females; mean age, 18.89 \pm 2.78$)$ with a score of 1,2 and 3 were diagnosed with mild $(n=21)$, moderate $(n=28)$ and severe $(n=19)$ spinal degeneration, respectively. Those who scored 0 exhibited no degeneration in the spine $(n=52$; 29 males and 23 females; mean age, 18.27 \pm 2.41 ) and served as controls. Comparisons of age $(\mathrm{t}=1.42 ; \mathrm{P}=0.16)$ and $\mathrm{sex}$ $\left(\chi^{2}=1.77 ; \mathrm{P}=0.18\right)$ between the patients and controls were not statistically significant. Of the 68 patients recruited, 43 patients were selected randomly to obtain their NPCs. These NPCs were then subdivided into three treatment groups as mentioned below.
Isolation and culture of NPCs. Degenerative NPCs were extracted from patients via puncture to the nucleus pulposus of the intervertebral disc. Normal NPCs were extracted from intervertebral disc tissue of the lesions following surgery for acute trauma. PBS was utilized to wash tissue in a sterile environment. NPCs were isolated (25), cut into sections (diameter, 2-3 mm), washed twice with PBS, digested with $0.2 \%$ protease at $37^{\circ} \mathrm{C}$ for $60 \mathrm{~min}$ and then digested with $0.02 \%$ type II collagenase overnight at $37^{\circ} \mathrm{C}$. After the detachment was terminated with Dulbecco modified Eagle medium Ham's F-12 (DMEM/F12; Thermo Fisher Scientific, Inc.), the NPCs were centrifuged at $201 \mathrm{x}$ g (5 min, 3 times) at room temperature. NPCs were collected, counted using cell-counting boards, and inoculated into a $25 \mathrm{~cm}$ culture flask at a density of $1 \times 10^{6}$ cells/ml. DMEM/F12 with $10 \%$ fetal bovine serum (FBS; Gibco; Thermo Fisher Scientific, Inc.) and $1 \%$ penicillin-streptomycin was subsequently added, and cells were incubated with $5 \% \mathrm{CO}_{2}$ at $37^{\circ} \mathrm{C}$ for $7-10$ days until cells covered the whole wall of the culture flask. During incubation, culture medium was refreshed every 3 days.

Cell Construction and transfection. pCR3.1-SOCS-3 and pCR3.1 were purchased from Invitrogen (Thermo Fisher Scientific, Inc., Waltham, MA, USA). NPCs were divided into the following four groups: The blank group (degenerative NPCs without transfection), the pCR3.1-SOCS-3 group (degenerative NPCs transfected with pCR3.1-SOCS-3), the pCR3.1 group (degenerative NPCs transfected with pCR3.1) and the normal group (normal NPCs without transfection). NPCs extracted and cultured from 42 SDD patients were allocated evenly into the following three groups: The blank group, the pCR3.1-SOCS-3 group and the pCR3.1 group, with 14 patients in each group. Passaged NPCs were inoculated into 24 -well plates at a density of $4 \times 10^{4}$ cells/well for $24 \mathrm{~h}$ at $37^{\circ} \mathrm{C}$. Cells were then transfected when the confluence of adherent cells reached $30-50 \%$. A total of $50 \mu 1$ Opti-MEM (Gibco; Thermo Fisher Scientific, Inc.) was used to dilute 20 pmol pCR3.1-SOCS-3 and pCR3.1, which were then gently mixed. Opti-MEM (50 $\mu \mathrm{l})$ was used to dilute $1 \mu \mathrm{l}$ liposome Lipofectamine 2000 (Invitrogen; Thermo Fisher Scientific, Inc.), which was mixed and incubated at room temperature for 5 min. Diluted pCR3.1 plasmids and liposome Lipofectamine 2000 were then mixed and incubated at room temperature for $20 \mathrm{~min}$. Each well received a mixture of liposome and vectors (100 $\mu \mathrm{l}$ ) and was added with DMEM/F12 (Gibco; Thermo Fisher Scientific, Inc.) with 10\% FBS (Gibco; Thermo Fisher Scientific, Inc.) and $1 \%$ penicillin-streptomycin. Cell culture plates were then incubated with $5 \% \mathrm{CO}_{2}$ at $37^{\circ} \mathrm{C}$ for $8 \mathrm{~h}$. The culture medium (DMEM/F12 with $2 \% \mathrm{FBS}$ ) was then replaced with DMEM-F12 cell culture medium with $10 \%$ FBS and incubated at $37^{\circ} \mathrm{C}$ for $48 \mathrm{~h}$.

Flow cytometry. After NPCs of each group were digested with trypsin and counted, they were resuspended in an Eppendorf (EP) tube at a density of $2 \times 10^{5}$ and incubated with $5 \mu$ Human TruStain FcX ${ }^{\mathrm{TM}}$ (Fc receptor blocking solution; BioLegend, Inc.) at room temperature for 5-10 min. Subsequently, cells were probed with PE-labeled monoclonal antibodies against TNF- $\alpha$ (1:20; cat. no. MABF352; Sigma-Aldrich; Merck KGaA) and FITC-labeled Annexin V (1:10; cat. no. ab63556; 
Abcam) at $4^{\circ} \mathrm{C}$ for $30 \mathrm{~min}$. After cells were centrifuged three times at $201 \mathrm{x} \mathrm{g} \mathrm{(5} \mathrm{min} \mathrm{each)} \mathrm{at} 37^{\circ} \mathrm{C}$, the aforementioned antibodies were washed with cell staining buffer. Flow cytometry was performed detection, and flowJo V10.0.7 software (FlowJo LLC) was applied for analysis.

ELISA. After cells in each group were cultured for 12,24 or $48 \mathrm{~h}$, cell supernatants were collected. TNF- $\alpha$ (cat. no. 555268; BD Pharmingen; BD Biosciences, San Jose, CA, USA) and leptin (SBJ-H1014, Nanjing SenBeiJia Biological Technology Co., Ltd., Nanjing, China) levels were detected using ELISA kits. The optical density (OD) value was determined at $450 \mathrm{~nm}$. TNF- $\alpha$ and leptin levels in samples were calculated using a standard curve.

Reverse transcription-quantitative polymerase chain reaction (RT-qPCR). NPCs from the blank, pCR3.1-SOCS-3, pCR3.1 and normal groups were collected. SOCS-3 levels were detected using RT-qPCR. Leptin at different concentrations $(0,1,2.5,5,10$ and $15 \mathrm{mM})$ were added to degenerative NPCs, and following $48 \mathrm{~h}$, cells were collected. The levels of leptin and SOCS-3 were examined following RT-qPCR. Total RNA was extracted from the NPCs of each group using TRIzol (cat. no. 1559602; Invitrogen; Thermo Fisher Scientific, Inc.) and then reversely transcribed to cDNA. First strand cDNA was synthesized using the High-Capacity cDNA reverse transcription kit (Applied Biosystems; Thermo Fisher Scientific, Inc.) according to the manufacturer's protocol. Subsequently, $1 \mu \mathrm{l}$ cDNA was added to $25 \mu \mathrm{l}$ of a quantitative fluorescence reaction system (SYBR Green Realtime PCR Master Mix; cat. no. QPK-201; Toyobo Life Science) containing, forward primer $(10 \mu \mathrm{M} ; 0.5 \mu \mathrm{l})$, reverse primer $(10 \mu \mathrm{M} ; 0.5 \mu \mathrm{l}), \mathrm{SYBR}$ Green Real-time PCR Master Mix (12.5 $\mu$ l; cat. no. QPK-201; Toyobo Life Science) and nuclease-free water (10.5 $\mu \mathrm{l})$. ABI 7500 PCR (Applied Biosystems; Thermo Fisher Scientific, Inc.) was used to analyze curves of PCR amplification and solubility. The primer sequences were as follows. Leptin forward, 5'-ATGCATTGGGAACCCTGTGCGG-3' and reverse, 5'-TGAGGTCCAGCTGCCACAGCATG-3'; SOCS-3 forward, 5'-CTACGCATCCAGTGTGAGGG-3' and reverse, 5'-TGA GTACACAGTCGAAGCGG-3'; $\beta$-actin forward 5'-TTGCCG ACAGGATGCAGAAGGA-3' and reverse, 5'-AGGTGGACA GCGAGGCCAGGAT-3'. The thermocycling conditions were as follows: $50^{\circ} \mathrm{C}$ for $2 \mathrm{~min}, 95^{\circ} \mathrm{C}$ for $2 \mathrm{~min}, 95^{\circ} \mathrm{C}$ for $15 \mathrm{sec}$, $60^{\circ} \mathrm{C}$ for $30 \mathrm{sec}$ and 40 cycles of amplification. Melting curves were then drawn using $95^{\circ} \mathrm{C}$ for $15 \mathrm{sec}, 60^{\circ} \mathrm{C}$ for $1 \mathrm{~min}, 85^{\circ} \mathrm{C}$ for $15 \mathrm{sec}$ and $60^{\circ} \mathrm{C}$ for $15 \mathrm{sec}$. Finally, results were obtained using the $2^{-\triangle \Delta C q}$ method (26), with $\beta$-actin as an internal reference. Experiments were repeated three times for each sample.

Western blot analysis. Recombined human leptin proteins at final concentrations of $0,1,2.5,5,10$ and $15 \mathrm{mM}$ were added to cells from each group in accordance with experimental requirements. Following $48 \mathrm{~h}$, cells from each group were collected. Changes in SOCS-3 levels were determined via western blotting $(27,28)$. Prepared protein samples $(20-60 \mu \mathrm{g} / \mathrm{l})$ were isolated via 12\% SDS-PAGE. Electrophoresis (80-120 V) was immediately terminated once bromophenol blue reached the bottom of the gel. Protein was then transferred onto nitrocellulose membranes for $1.5 \mathrm{~h}$ at $100 \mathrm{~V}$. After washing with PBS containing $0.5 \%$ Tween 20 (PBST), the membranes were blocked in $5 \%$ skimmed milk at room temperature for $2 \mathrm{~h}$. The following primary antibodies were then added and incubated at $4^{\circ} \mathrm{C}$ overnight: GAPDH (cat. no. ab37168; 1:1,000), SOCS-3 (cat. no. ab16030; 1:1,000), leptin receptor (cat. no. ab5593; 1:2,000), leptin (cat. no. ab3583; 1:500) and phosphorylated leptin receptor (cat. no. PA5-64634; 1:5,000). Membranes were washed four times with PBST (each, $10 \mathrm{~min}$ ) and incubated with secondary antibodies (horseradish peroxidase-conjugated goat anti-rabbit IgG (cat. no. ab97051; $1: 2,000)$ at room temperature for $2 \mathrm{~h}$ on a shaking table (HS 501 digita; IKA-Werke, Staufen, Germany). All antibodies were purchased from Abcam. Membranes were then washed four times with PBST (each, $10 \mathrm{~min}$ ) and developed using a chemiluminescence (ECL) fluorescent detection kit (cat. no. BB-3501; GE Healthcare, Chicago, IL, USA). ImageJ 1.46r software (National Institutes of Health, Bethesda, MD, USA) was utilized to quantitatively analyze the molecular weight and net optical density value of target bands, with GAPDH used as a reference.

Statistical analysis. SPSS 20.0 software (IBM Corp., Armonk, NY, USA) was utilized for data analysis. Data are expressed as the mean \pm standard deviation. Normal distribution and variance homogeneity were tested for all the data. The statistical significance of data with normal distribution and equal variance between two groups were compared using an unpaired t-test. Comparisons among multiple groups were analyzed using one-way analysis of variance or repeated measurement analysis of variance, followed by a Tukey's post-hoc test. Data with a skewed distribution or heterogeneity of variance were compared using a rank sum test. The correlation between SOCS-3 and phosphorylated leptin receptor levels were analyzed using a Pearson's correlation test. $\mathrm{P}<0.05$ was considered to indicate a statistically significant difference.

\section{Results}

Overexpression of SOCS-3 decreases TNF- $\alpha$ levels in degenerative NPCs. To assess the expression of TNF- $\alpha$ in degenerative NPCs transfected with SOCS -3 , flow cytometry was performed. The number of TNF- $\alpha$ positive cells and the MFI of the blank group were significantly higher than that of the normal group $(\mathrm{P}<0.05)$. TNF- $\alpha$ was overexpressed in degenerative NPCs and underexpressed in normal NPCs. Following degenerative NPC transfection, the number of TNF- $\alpha$ positive cells and the MFI of the pCR3.1-SOCS-3 group significantly decreased when compared with the pCR3.1 group $(\mathrm{P}<0.05$; Fig. 1). These data indicate that SOCS-3 overexpression results in a significant reduction of TNF- $\alpha$ in degenerative NPCs.

Overexpression of SOCS-3 decreases levels of TNF- $\alpha$ and leptin in degenerative NPCs. To assess the effect of SOCS-3 on TNF- $\alpha$ and leptin in degenerative NPCs, ELISA was performed. As presented in Fig. 2, TNF- $\alpha$ and leptin levels were significantly higher in the blank group compared with the normal group at 12, 24 and $48 \mathrm{~h}(\mathrm{P}<0.05)$. Following the transfection of degenerative NPCs, levels of TNF- $\alpha$ and leptin were significantly decreased in the pCR3.1-SOCS-3 group compared with the pCR3.1 group $(\mathrm{P}<0.05)$, indicating that 

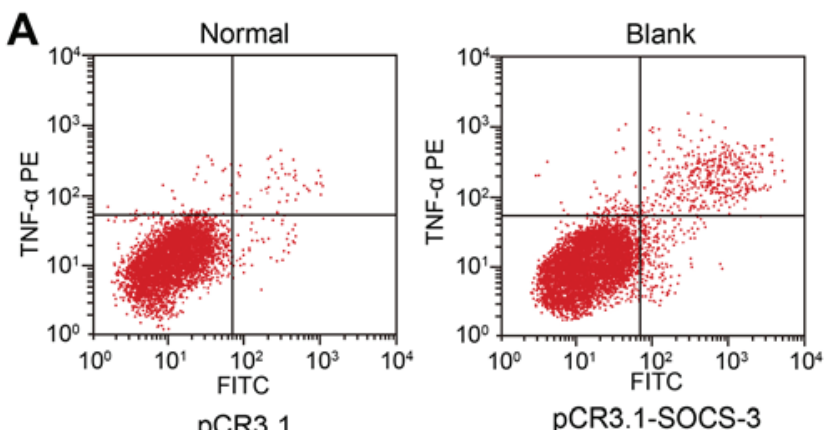

B
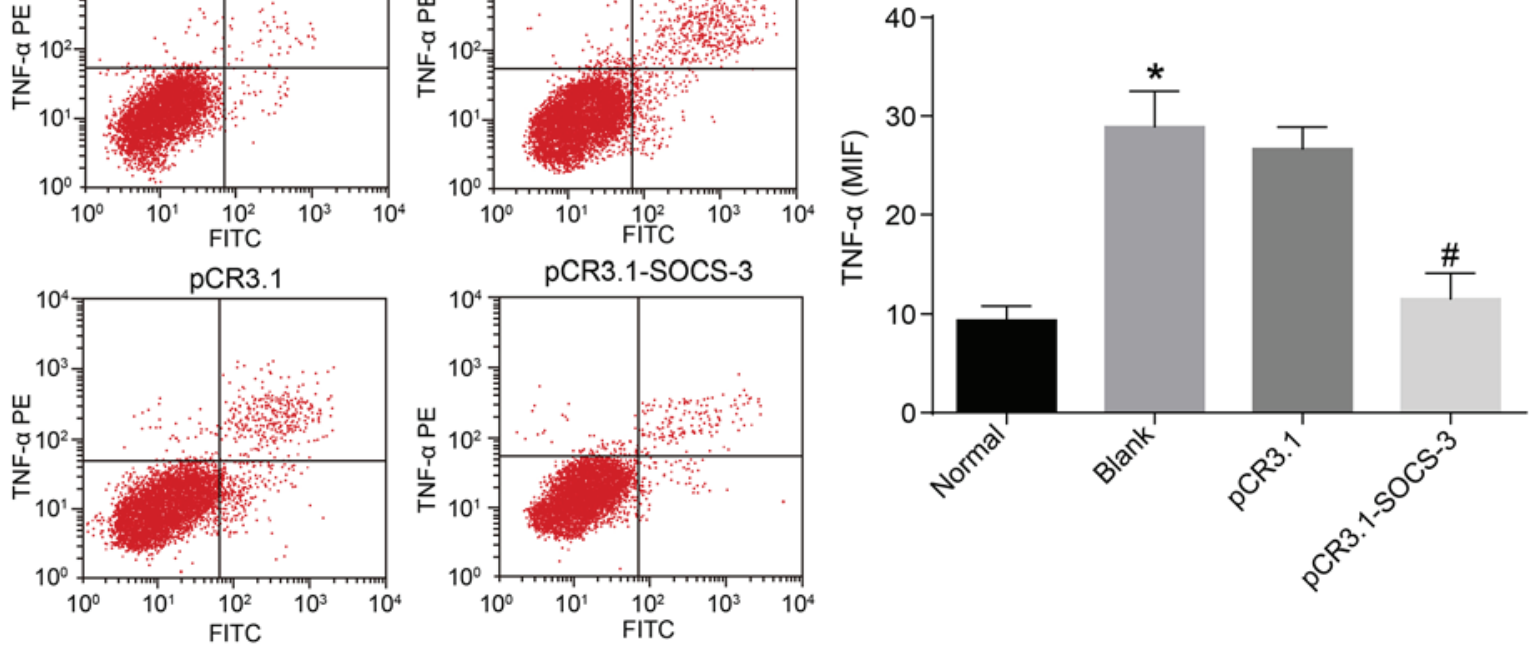

Figure 1. Overexpression of SOCS-3 reduces TNF- $\alpha$ and MFI in degenerative NPCs. (A) TNF- $\alpha$ positive cells detected using flow cytometry. (B) MFI of TNF- $\alpha$ in NPCs. Each experiment was repeated three times. Data are presented as the mean \pm standard deviation $(n=14)$. "P<0.05 vs. the normal group; ${ }^{*} \mathrm{P}<0.05$ vs. the pCR3.1 group. SOCS-3, suppressor of cytokine signaling 3; TNF- $\alpha$, tumor necrosis factor- $\alpha$; MFI, mean fluorescence intensity; NPC, nucleus pulposus cell; PE, phycoerythrin; FITC, fluorescein isothiocyanate.
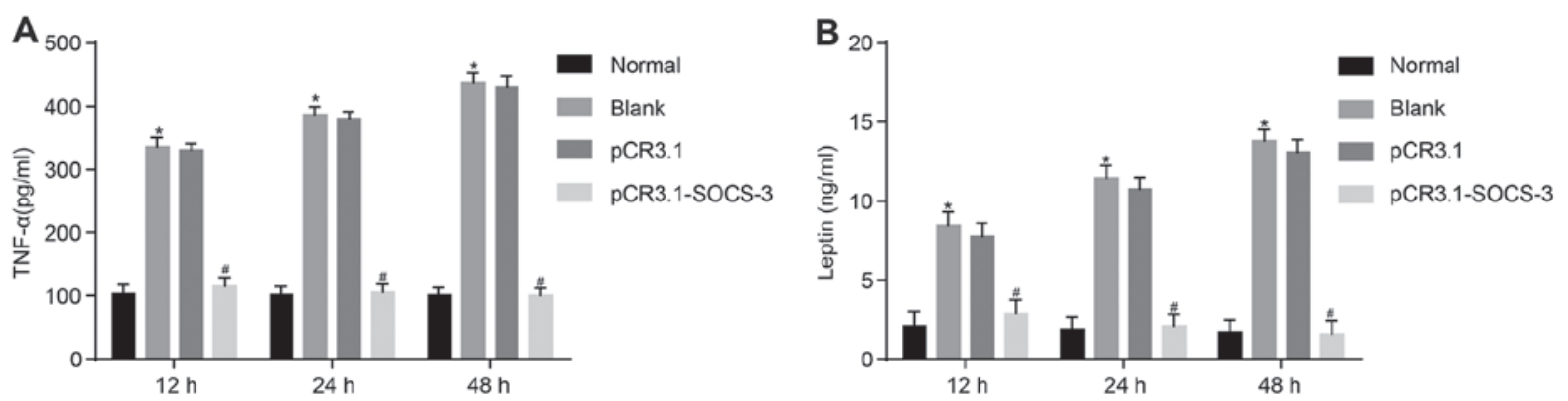

Figure 2. Overexpression of SOCS-3 reduces levels of TNF- $\alpha$ and leptin in degenerative NPCs. Levels of (A) TNF- $\alpha$ and (B) leptin were detected in the supernatant of NPCs using ELISA. Each experiment was repeated three times. Data are expressed as the mean \pm standard deviation $(\mathrm{n}=14)$. ${ }^{*} \mathrm{P}<0.05 \mathrm{vs}$. the normal group; "P<0.05 vs. the pCR3.1 group. SOCS-3, suppressor of cytokine signaling 3; TNF- $\alpha$, tumor necrosis factor- $\alpha$; NPC, nucleus pulposus cell.

levels of TNF- $\alpha$ and leptin were increased in degenerative NPCs without treatment and remained relatively low in normal NPCs. The results revealed that the overexpression of SOCS-3 reduces the level of TNF- $\alpha$ and leptin in degenerative NPCs.

Leptin induces SOCS-3 mRNA levels. To assess the mechanism and function of SOCS-3 and leptin in NPCs, their expression was determined using RT-qPCR. SOCS-3 was revealed to be poorly expressed in degenerative NPCs compared with normal NPCs $(\mathrm{P}<0.05)$. SOCS-3 mRNA levels in the pCR3.1-SOCS-3 group were markedly higher than those in the pCR3.1 group $(\mathrm{P}<0.05)$. However, SOCS-3 mRNA levels in the pCR3.1 group were not significantly different from those in the blank group (Fig. 3A and B). Following degenerative NPC culture with increasing concentrations of leptin for $48 \mathrm{~h}$, SOCS-3 mRNA was induced in a concentration-dependent manner (Fig. 3C and D).

Leptin increases SOCS-3 protein levels in a concentrationdependent manner. The ability of SOCS-3 to influence the biological function of leptin in NPCs was assessed. The leptin receptor itself does not possess tyrosine kinase activity and therefore is phosphorylated by leptin only. Different concentrations of leptin were added to degenerative NPCs to induce the upregulation of SOCS-3 for $48 \mathrm{~h}$. Leptin expression and leptin receptor phosphorylation was determined using western blotting. The results demonstrated that SOCS-3 protein levels increased gradually with increasing concentrations of leptin in degenerative NPCs (Fig. 4A). However, the extent of leptin receptor phosphorylation (Fig. 4B) gradually decreased with increasing leptin concentrations. A Pearson's correlation analysis was performed to assess the correlation between SOCS-3 and phosphorylated leptin receptor levels. The results revealed that phosphorylated leptin receptor levels were negatively correlated with SOCS-3 levels (Fig. 4C). Thus, leptin induces SOCS-3 in a concentration-dependent manner.

\section{Discussion}

Although SDD is rare in pediatric and adolescent populations, those affected experience a reduced quality of life and 

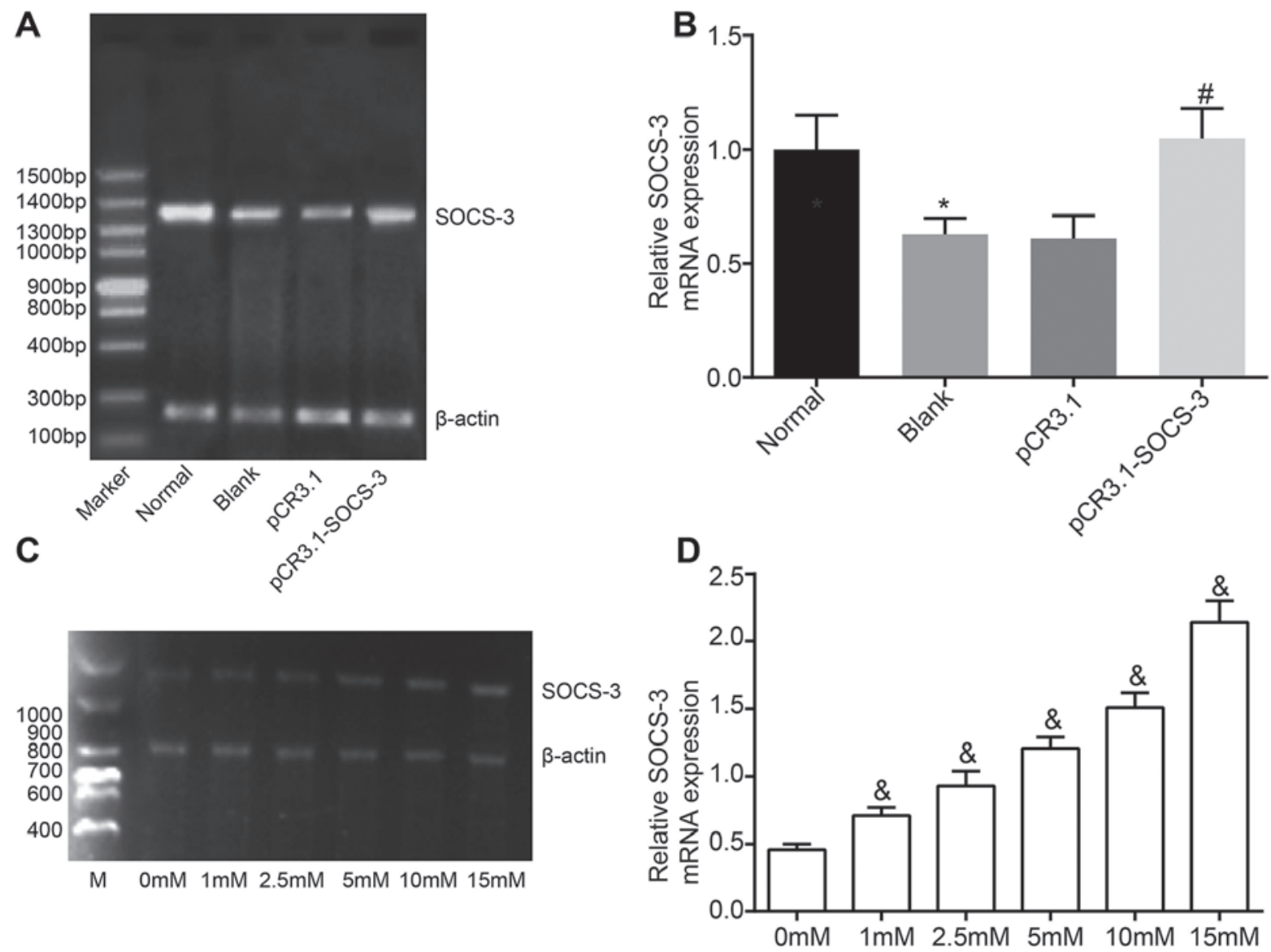

Figure 3. Leptin induces SOCS-3 mRNA in a concentration-dependent manner. (A) Electrophoretogram and (B) quantitative gray values of SOCS-3 in NPCs. (C) Electrophoretogram and (D) quantitative gray values of SOCS-3 treated with different concentration of leptin in degenerative NPCs. Each experiment was repeated three times and data are expressed as the mean \pm standard deviation. ${ }^{*} \mathrm{P}<0.05$ vs. the control group; ${ }^{\prime} \mathrm{P}<0.05$ vs. the pCR 3.1 group; ${ }^{\circledR} \mathrm{P}<0.05$ vs. the 0 mM group. SOCS-3, suppressor of cytokine signaling 3; NPC, nucleus pulposus cell.

A
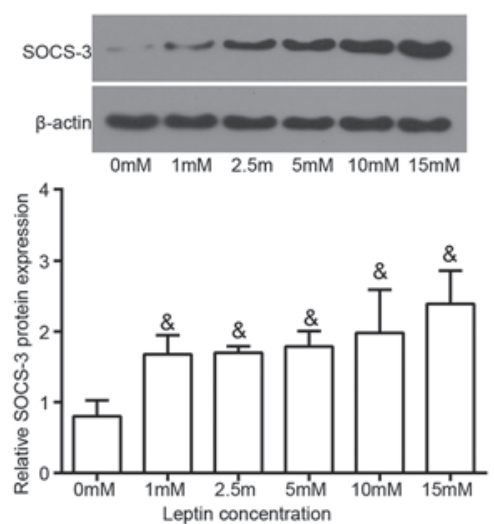

B
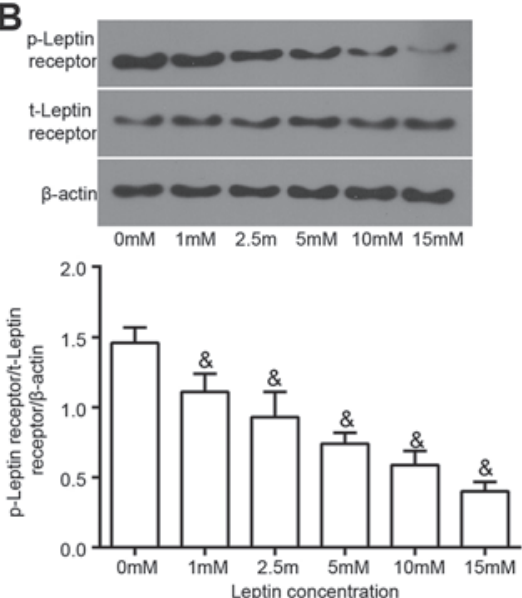

C

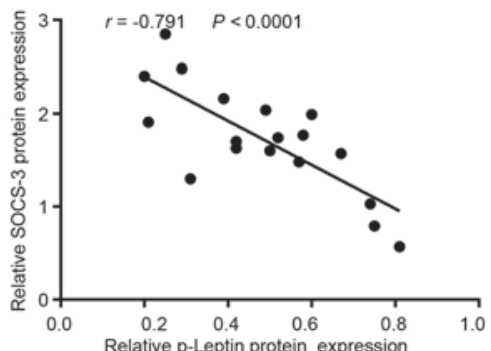

Figure 4. Leptin increases SOCS-3 levels and decreases leptin receptor phosphorylation in a concentration-dependent manner. (A) SOCS-3 protein levels were assessed and quantified following the treatment with different concentrations of exogenous leptin. (B) Extent of leptin receptor phosphorylation, with gray value analysis. (C) A Spearman's rank correlation analysis was performed on SOCS-3 and leptin phosphorylation levels. Each experiment was repeated three times and data are expressed as the mean \pm standard deviation. ${ }^{\circledR} \mathrm{P}<0.05$ vs. the $0 \mathrm{mM}$ group. SOCS -3 , suppressor of cytokine signaling 3 ; p-leptin, phosphorylated leptin; t-leptin, total leptin.

suffering (29). The SOCS family [comprised of eight genes (SOCS1 to SOCS7 along with CIS) that share similar structures], function as negative mediators of cytokine signaling and serve a critical role in the immune system $(30,31)$. The current study assessed the effect of SOCS-3 on adolescent SDD and its association with leptin and TNF- $\alpha$. The results demonstrated 
that the overexpression of SOCS-3 reduced leptin levels and inhibited TNF- $\alpha$, providing a theoretical foundation for SDD treatment in adolescents.

Initially, the present study revealed that the number of TNF- $\alpha$ positive cells in degenerative NPCs was significantly higher compared with normal NPCs, indicating that TNF- $\alpha$ is overexpressed in the former. Furthermore, the number of TNF- $\alpha$ positive cells in degenerative NPCs transfected with pCR3.1-SOCS-3 were reduced compared with those transfected with pCR3.1, indicating that the overexpression of SOCS-3 decreases TNF- $\alpha$ levels. TNF- $\alpha$ is a proinflammatory cytokine that contributes to inflammation-induced disease pathology, while SOCS proteins function as suppressors of cytokine signaling and mediators of inflammation (23). A previous study determined the prominent role of SOCS-3 in herniated lumbar disc degeneration by inhibiting the JAK/STAT3 signaling pathway (14). SOCS-3 also exhibits anti-inflammatory and anti-proliferation effects in osteoarthritis by downregulating levels of nuclear factor- $\kappa \mathrm{B}$ and cyclooxygenase 2 (15). Dai et al (32) demonstrated that the upregulation of SOCS-3 expression results in reduced TNF- $\alpha$ levels and the regulation of Kallikrein-binding protein. Furthermore, Collino et al (33) determined that SOCS-3 restoration leads to a decreased local inflammatory response by suppressing levels of TNF- $\alpha$ in patients with acute kidney injury (33).

The ELISA results of the current study demonstrated that levels of TNF- $\alpha$ and leptin at 12, 24 and $48 \mathrm{~h}$ in degenerative NPCs were significantly higher than in normal NPCs. Following transfection with pCR3.1, levels of TNF- $\alpha$ and leptin in the pCR3.1-SOCS-3 group decreased to a greater extent than in the pCR3.1 group. This indicates that TNF- $\alpha$ and leptin are overexpressed in degenerative NPCs free of any treatment and underexpressed in normal NPCs. Furthermore, overexpressed SOCS-3 was determined to decrease TNF- $\alpha$ and leptin levels in degenerative NPCs. A previous study by Ohtori et al (34) revealed that more TNF- $\alpha$ immune-reactive cells were observed in the nucleus pulposus from adolescent patients with lumbar disc herniation with compared with adolescent patients with nonpainful scoliosis, indicating that TNF- $\alpha$ may be associated with disc degeneration and pain in adolescent patients with lumbar disc herniation. TNF- $\alpha$ was also revealed to initiate early stage disc degeneration, which indicates that it may be an early pathogenetic factor in disc degeneration (35). In addition, Wang et al (36) determined that leptin levels were elevated in rat models of femoral fracture and traumatic spinal cord injury.

In addition to the inhibitory effect of SOCS-3 on leptin, the results indicated that leptin induces SOCS-3 expression. SOCS-3 has been reported to negatively regulate leptin in patients with osteoarthritis (37). Yang et al (38) also demonstrated that SOCS-3 negatively regulates leptin signaling in the hypothalamus and that leptin induces SOCS-3, thus preventing leptin signaling and indicating that SOCS-3 functions as a regulator of leptin sensitivity (38). Furthermore, a previous study determined that high-dose leptin triggers the increased expression of SOCS-3 in people that are obese (39).

In conclusion, the present study revealed that the upregulated expression of SOCS-3 may be a novel treatment target for SDD, as SOCS-3 overexpression may alleviate SDD by reducing leptin expression and TNF- $\alpha$ levels. However, the relatively small sample size in the current study may have influenced the results obtained. Thus, further studies with larger sample sizes should be performed to explore the protective value of SOCS-3 in adolescent patients with SDD. It is anticipated that with a further understanding of SDD, SOCS-3 and leptin may be a promising target for the prevention and treatment of SDD in adolescents.

\section{Acknowledgements}

Not applicable.

\section{Funding}

No funding was received.

\section{Availability of data and materials}

The datasets used and/or analyzed during the present study are available from the corresponding author on reasonable request.

\section{Authors' contributions}

XT and JD designed the current study, JD and HS collected the data. XT and JD performed data analyses and produced the initial draft of the manuscript. All authors have read and approved the final submitted manuscript.

\section{Ethics approval and consent to participate}

The experimental protocols of the present study were approved by the Ethical Committee of Huai'an First People's Hospital (Huai'an, China). All patients provided signed informed consent.

\section{Patient consent for publication}

Not applicable.

\section{Competing interests}

The authors declare they have no competing interests.

\section{References}

1. Brinjikji W, Luetmer PH, Comstock B, Bresnahan BW, Chen LE, Deyo RA, Halabi S, Turner JA, Avins AL, James K, et al: Systematic literature review of imaging features of spinal degeneration in asymptomatic populations. AJNR Am J Neuroradiol 36: 811-816,2015.

2. Matsumoto M, Okada E, Ichihara D, Watanabe K, Chiba K, Toyama Y, Fujiwara H, Momoshima S, Nishiwaki Y, Hashimoto T, et al: Age-related changes of thoracic and cervical intervertebral discs in asymptomatic subjects. Spine (Phila $\mathrm{Pa}$ 1976) 35: 1359-1364, 2010.

3. Lao LF, Zhong GB, Li QY and Liu ZD: Kinetic magnetic resonance imaging analysis of spinal degeneration: A systematic review. Orthop Surg 6: 294-299, 2014.

4. Goel A: Facet distraction spacers for treatment of degenerative disease of the spine: Rationale and an alternative hypothesis of spinal degeneration. J Craniovertebr Junction Spine 1: 65-66, 2010.

5. Hey HW and Hee HT: Lumbar degenerative spinal deformity: Surgical options of PLIF, TLIF and MI-TLIF. Indian J Orthop 44: 159-162, 2010. 
6. Takatalo J, Karppinen J, Näyhä S, Taimela S, Niinimäki J, Blanco Sequeiros R, Tammelin T, Auvinen J and Tervonen O: Association between adolescent sport activities and lumbar disk degeneration among young adults. Scand J Med Sci Sports 27: 1993-2001, 2017.

7. Wang H, Cheng J, Xiao H, Li C and Zhou Y: Adolescent lumbar disc herniation: Experience from a large minimally invasive treatment centre for lumbar degenerative disease in Chongqing, China. Clin Neurol Neurosurg 115: 1415-1419, 2013.

8. Daniels DJ, Luo TD, Puffer R, McIntosh AL, Larson AN, Wetjen NM and Clarke MJ: Degenerative changes in adolescent spines: A comparison of motocross racers and age-matched controls. J Neurosurg Pediatr 15: 266-271, 2015.

9. Omair A, Holden M, Lie BA, Reikeras O and Brox JI: Treatment outcome of chronic low back pain and radiographic lumbar disc degeneration are associated with inflammatory and matrix degrading gene variants: A prospective genetic association study. BMC Musculoskelet Disord 14: 105, 2013.

10. Ren Y and Young W: Managing inflammation after spinal cord injury through manipulation of macrophage function. Neural Plast 2013: 945034, 2013

11. Carow B and Rottenberg ME: SOCS3, a Major regulator of infection and inflammation. Front Immunol 5: 58, 2014.

12. Park KW, Lin CY and Lee YS: Expression of suppressor of cytokine signaling-3 (SOCS3) and its role in neuronal death after complete spinal cord injury. Exp Neurol 261: 65-75, 2014.

13. Park KW, Lin CY, Li K and Lee YS: Effects of reducing suppressors of cytokine signaling-3 (SOCS3) expression on dendritic outgrowth and demyelination after spinal cord injury. PLoS One 10: e0138301, 2015.

14. Osuka K, Usuda N, Aoyama M, Yamahata H, Takeuchi M, Yasuda Mand Takayasu M: Expression of the JAK/STAT3/SOCS3 signaling pathway in herniated lumbar discs. Neurosci Lett 569 55-58, 2014.

15. Gui T, He BS, Gan Q and Yang C: Enhanced SOCS3 in osteoarthiritis may limit both proliferation and inflammation. Biotech Histochem 92: 107-114, 2017.

16. Dreier R: Hypertrophic differentiation of chondrocytes in osteoarthritis: The developmental aspect of degenerative joint disorders. Arthritis Res Ther 12: 216, 2010.

17. Babon JJ and Nicola NA: The biology and mechanism of action of suppressor of cytokine signaling 3. Growth Factors 30 207-219, 2012

18. Lubis AR, Widia F, Soegondo S and Setiawati A: The role of SOCS-3 protein in leptin resistance and obesity. Acta Med Indones 40: 89-95, 2008

19. Garner M, Alshameeri Z and Khanduja V: Osteoarthritis: Genes, nature-nurture interaction and the role of leptin. Int Orthop 37: 2499-2505, 2013.

20. Vuolteenaho K, Koskinen A and Moilanen E: Leptin-a link between obesity and osteoarthritis. Applications for prevention and treatment. Basic Clin Pharmacol Toxicol 114: 103-108, 2014.

21. Mutabaruka MS, Aoulad Aissa M, Delalandre A, Lavigne M and Lajeunesse D: Local leptin production in osteoarthritis subchondral osteoblasts may be responsible for their abnormal phenotypic expression. Arthritis Res Ther 12: R20, 2010.

22. Johnson ZI, Schoepflin ZR, Choi H, Shapiro IM and Risbud MV: Disc in flames: Roles of TNF-alpha and IL-1 $\beta$ in intervertebra disc degeneration. Eur Cell Mater 30: 104-116, 2015.

23. Collins AS, Ahmed S, Napoletano S, Schroeder M, Johnston JA, Hegarty JE, O'Farrelly C and Stevenson NJ: Hepatitis C virus (HCV)-induced suppressor of cytokine signaling (SOCS) 3 regulates proinflammatory TNF- $\alpha$ responses. J Leukoc Biol 96 $255-263,2014$
24. Poiraudeau S, Monteiro I, Anract P, Blanchard O, Revel M and Corvol MT: Phenotypic characteristics of rabbit intervertebral disc cells. Comparison with cartilage cells from the same animals. Spine (Phila Pa 1976) 24: 837-844, 1999.

25. Chelberg MK, Banks GM, Geiger DF and Oegema TR Jr: Identification of heterogeneous cell populations in normal human intervertebral disc. J Anat 186: 43-53, 1995.

26. Livak KJ and Schmittgen TD: Analysis of relative gene expression data using real-time quantitative PCR and the 2(-Delta Delta C(T)) method. Methods 25: 402-408, 2001.

27. Yu J, Ryan DG, Getsios S, Oliveira-Fernandes M, Fatima A and Lavker RM: MicroRNA-184 antagonizes microRNA-205 to maintain SHIP2 levels in epithelia. Proc Natl Acad Sci USA 105: 19300-19305, 2008.

28. Yu J, Peng H, Ruan Q, Fatima A, Getsios S and Lavker RM: MicroRNA-205 promotes keratinocyte migration via the lipid phosphatase SHIP2. FASEB J 24: 3950-3959, 2010.

29. Kumar R, Kumar V, Das NK, Behari S and Mahapatra AK: Adolescent lumbar disc disease: Findings and outcome. Childs Nerv Syst 23: 1295-1299, 2007.

30. Lindemann C, Hackmann O, Delic S, Schmidt N, Reifenberger G and Riemenschneider MJ: SOCS3 promoter methylation is mutually exclusive to EGFR amplification in gliomas and promotes glioma cell invasion through STAT3 and FAK activation. Acta Neuropathol 122: 241-251, 2011.

31. Kershaw NJ, Murphy JM, Liau NP, Varghese LN, Laktyushin A, Whitlock EL, Lucet IS, Nicola NA and Babon JJ: SOCS3 binds specific receptor-JAK complexes to control cytokine signaling by direct kinase inhibition. Nat Struct Mol Biol 20: 469-476, 2013.

32. Dai Z, Lu L, Yang Z, Mao Y, Lu J, Li C, Qi W, Chen Y, Yao Y, $\mathrm{Li}$ L, et al: Kallikrein-binding protein inhibits LPS-induced TNF- $\alpha$ by upregulating SOCS3 expression. J Cell Biochem 114: $1020-1028,2013$

33. Collino M, Benetti E, Miglio G, Castiglia S, Rosa AC, Aragno $\mathrm{M}$, Thiemermann $\mathrm{C}$ and Fantozzi R: Peroxisome proliferator-activated receptor $\beta / \delta$ agonism protects the kidney against ischemia/reperfusion injury in diabetic rats. Free Radic Biol Med 50: 345-353, 2011

34. Ohtori S, Inoue G, Eguchi Y, Orita S, Takaso M, Ochiai N, Kishida S, Kuniyoshi K, Aoki Y, Nakamura J, et al: Tumor necrosis factor- $\alpha$-immunoreactive cells in nucleus pulposus in adolescent patients with lumbar disc herniation. Spine (Phila Pa 1976) 38: 459-462, 2013.

35. Kang R, Li H, Rickers K, Ringgaard S, Xie L and Bünger C: Intervertebral disc degenerative changes after intradiscal injection of TNF- $\alpha$ in a porcine model. Eur Spine J 24: 2010-2016, 2015.

36. Wang L, Tang X, Zhang H, Yuan J, Ding H and Wei Y: Elevated leptin expression in rat model of traumatic spinal cord injury and femoral fracture. J Spinal Cord Med 34: 501-509, 2011.

37. Vuolteenaho K, Koskinen A, Moilanen T and Moilanen E: Leptin levels are increased and its negative regulators, SOCS-3 and $\mathrm{sOb}-\mathrm{R}$ are decreased in obese patients with osteoarthritis: A link between obesity and osteoarthritis. Ann Rheum Dis 71: 1912-1913, 2012

38. Yang Z, Hulver M, McMillan RP, Cai L, Kershaw EE, Yu L, Xue B and Shi H: Regulation of insulin and leptin signaling by muscle suppressor of cytokine signaling 3 (SOCS3). PLoS One 7: e47493, 2012

39. Teran-Cabanillas E and Hernandez J: Role of Leptin and SOCS3 in inhibiting the type I interferon response during obesity. Inflammation 40: 58-67, 2017. 